\title{
ON THE FIXED POINT SET \\ OF A COMPACT TRANSFORMATION GROUP WITH SOME APPLICATIONS TO COMPACT MONOIDS
}

BY

\author{
KARL HEINRICH HOFMANN AND MICHAEL MISLOVE(1)
}

\begin{abstract}
Under various special additional hypotheses we prove that the fixed point set of the group of inner automorphisms of a compact connected monoid with zero is connected.
\end{abstract}

We prove a result which implies the following theorem: If a compact group $G$ acts on an arcwise connected space $X$ with at least one fixed point, then an orbit $x G$ is a retract of $X$ if and only if it is singleton (i.e., $x$ is a fixed point). When applied with the $n$-dimensional euclidean unit ball $X$ and $S O(n)$ this implies Brouwer's fixed point theorem. A crucial step in the proof of this result is a proof of the following: If $G$ is a compact group and $K_{1} \leqslant K_{2} \leqslant G$ are closed subgroups, then any continuous function $G / K_{1} \rightarrow G / K_{2}$ which is homotopic to the natural map is surjective (and indeed this holds for certain projective limits of manifolds in place of the $G / K_{j}$ ).

We apply this result to obtain a partial solution to a problem in transformation groups which belongs to the following rather prevalent type of question: If $G$ is a compact group acting on a compact acyclic space $X$ (relative to cohomology over some fixed coefficient module), is then the space Fix $(G, X)$ of fixed points acyclic? The answer, in general, is no. If $G$ is connected and abelian and the coefficient module is a field of characteristic zero then the answer is affirmative according to theorems of Conner and Borel. If $G$ is a finite cyclic group of $p$-power order and the coefficient module is a field of characteristic $p$, then the affirmative answer arises from Smith theory (or likewise from the Conner-Borel technique). The construction of appropriate examples has shown that further answers are not to be expected on a purely transformation group theoretical basis.

However, if $X$ is a compact connected monoid with zero (imagine, for

Received by the editors January 19,1973 and, in revised form, January 4, 1974. AMS (MOS) subject classifications (1970). Primary 22A15, 22C05, 54H1 5.

Key words and phrases. Compact semigroups, compact transformation groups, fixed points, centralizer.

(1) Both authors supported by NSF Grant GP28655.

Copyright $\odot$ 1975, American Mathematical Society 
instance, the quaternion unit ball as a rather trivial illustration), then $X$ is acyclic over all coefficient groups. If a compact group $G$ acts as a group of automorphisms on $X$ then we add a new ingredient to the general question above, and indeed the following conjecture is amply supported by all of the available evidence:

Fixed Point Conjecture. The fixed point set Fix $(G, X)$ of a compact group of automorphisms of a compact connected monoid $X$ with zero is connected (hence, being a monoid, is acyclic relative to Čech cohomology over any coefficient module).

In past work on the problem it has been useful to consider an equivalent version of the same conjecture which is phrased in more semigroup theoretical terms (which one might test for the quaternion unit ball):

Centralizing Conjecture. If $S$ is a compact connected monoid with zero and $H$ the group of units then the centralizer $Z(H, S)=\{s \in S \mid s h=h s$ for all $h \in H\}$ of $H$ in $S$ is connected.

In view of existing examples these conjectures definitely do not form an exclusive transformation group theoretical problem. However, no progress towards a solution has ever been made without some applications of transformation group theoretical methods. The same holds for the present paper in which we offer partial contributions to the centralizing conjecture. The first part of the paper is purely group and transformation group theoretical leading to the results we mentioned initially. In order to apply them we prove some transformation semigroup theoretical results in $\S 2$ which are elementary by comparison. In $\S 3$ we bring transformation groups and transformation semigroups together to prove the following centralizing result:

THE $H \times H$-THEOREM (SPECIAL VERSION). Let $S$ be a compact connected monoid with zero and no other idempotents and suppose that the space of cosets $H s H$ (which is an orbit space of the action of $H \times H$ on $S$ given by $(h, k) \cdot s$ $\left.=h s k^{-1}\right)$ is homeomorphic to $[0,1]$. Then the centralizing conjecture holds.

(One observes that the example of the quaternion unit ball is covered by this theorem.) The result is expanded in $\S 4$; we present various generalizations and applications of the technique, and in $\$ 5$ we give some illustrations shedding light on the limitations of the methods. The sections pertaining to the semigroup applications require some background knowledge in compact semigroup theory (such as Green's relations and the chaining process) for which [6] is a convenient reference for our purposes.

The $H \times H$ theorem above was formulated in [6] on p.177. The proof was 
discovered to be faulty by J. H. Carruth in 1971 . The proof presented here is based on different ideas, using among other things a result of the authors [5] which is also based partially on transformation group theoretical and partially on semigroup theoretical arguments. It was believed that the peripherality of the units in a compact connected monoid would be essential for a proof of the $\mathrm{H} \times \boldsymbol{H}$ theorem, but no such consideration enters the present proof.

The authors would like to take this opportunity to acknowledge the efforts of J. H. Carruth on this problem. His discovery of the gap in the proof of the $H \times H$ theorem in [6] provided the impetus not only for this work, but also for the results in [5]. His comments and criticisms both in informal discussions as well as in his role as referee of this paper have contributed greatly to its final form.

\section{A transformation group theoretical generalization of Brouwer's fixed} point theorem. Brouwer's fixed point theorem (stating that every continuous self-map of a closed $n$-cell has a fixed point) is, by a familiar and very elementary geometric argument, equivalent to the following assertion:

THEOREM 1.0. The sphere $S^{n-1}$ is not a retract of the cell $C^{n}$.

Transformation groups have to do with this result insofar as the special orthogonal group $S O(n)$ operates on the unit ball $C^{n}$ in $\mathbf{R}^{n}$ under rotations in such a fashion that

(i) $0 \in C^{n}$ is a fixed point,

(ii) $S^{n-1}$ is an orbit,

(iii) The orbit space $C^{n} / S O(n)$ is homeomorphic to the unit interval $[0,1]$.

This section is reserved for the proof of the following generalization of Theorem 1.0 above:

THEOREM 1.1. Let $G$ be a compact group acting on a space $X$ (on the right) in such a fashion that the following hypotheses are satisfied:

(1) There is at least one fixed point $x_{0} \in X$.

(2) The orbit $x G$ is a retract of $X$.

(3) The orbits $\left\{x_{0}\right\}$ and $x G$ are in the same arc component of the orbit space $X / G$.

Then $x$ is a fixed point.

We begin by recording the arc lifting lemma by Montgomery and Yang.

LEMMA 1.2. If $G$ is a compact Lie group operating on a compact space $X$ such that $X / G$ is an arc (homeomorphic to $[0,1]$ ), then there is a global cross section $\sigma: X / G \rightarrow X$ for the orbit map $p: X \rightarrow X / G$. 
(For the proof see [8].)

LEMMA 1.3. Lemma 1.2 maintains for an arbitrarily compact group in place of a compact Lie group, i.e. "every arc lifts".

(The technique discussed in [6, Lemma 1.12, p. 317] is immediately adaptable to cover the transition from the Lie group case of Lemma 1.2 to the case of an arbitrary compact group.)

LemMA 1.4. Let $G$ be a compact group acting on a compact space $X$ with at least one fixed point $x_{0}$. Suppose that the two orbits $\left\{x_{0}\right\}$ and $x G$ are in the same arc component of $X / G$. Then the map $f: G \rightarrow X$ defined by $f(g)=x g$ is homotopic to a constant map.

PROOF. Since $p\left(x_{0}\right)$ and $p(x)$ are in the same arc component of $X / G$, there is an injective continuous map $a:[0,1] \rightarrow X / G$ with $a(0)=p(x), a(1)=p\left(x_{0}\right)$. If we apply the Arc Lifting Lemma 2.1. to the $G$-space $X^{\prime}=p^{-1}(a([0,1]))$ we obtain a cross section $\sigma^{\prime}: a([0,1]) \rightarrow X^{\prime}$ for the orbit map $p^{\prime}=p \mid X^{\prime}: X^{\prime} \rightarrow$ $X^{\prime} / G$. Define $s:[0,1] \rightarrow X$ by $s(r)=\sigma^{\prime}(a(r))$. Then $p(s(0))=p\left(\sigma^{\prime}(a(0))\right)=$ $1_{X^{\prime}}(p(x))=p(x)$, whence $s(0) \in x G$. We find therefore an $h \in G$ with $s(0) h=$ $x$. Similarly, $p(s(1)) \in x_{0} G=\left\{x_{0}\right\}$; thus $s(1)=x_{0}$ and also $s(1) h=x_{0}$. Finally we define $F: G \times[0,1] \rightarrow X$ by $F(g, r)=s(r) h g$. Then $F$ is continuous and satisfies $F(g, 0)=s(0) h g=x g=f(g)$ and $F(g, 1)=s(1) h g=x_{0} g=x_{0}$ for all $g \in G$, and so $F$ is the desired homotopy.

Lemma 1.5. Suppose that $f: G \rightarrow X$ is a continuous map of topological spaces with $f(G) \subseteq Y \subseteq X$. If $Y$ is a retract of $X$ and $f$ is homotopic to a constant in $X$, then $f$ is homotopic to a constant in $Y$.

Proof. This is straightforward: If $r: X \rightarrow Y$ is a continuous retraction (i.e., $r(y)=y$ for $y \in Y$ ) and $F: G \times[0,1] \rightarrow X$ a homotopy deforming $f$ into a constant, then $r F: G \times[0,1] \rightarrow Y$ is a homotopy deforming $f$ into a constant.

LEMMA 1.6. Let $G$ be a compact group, $K$ a closed subgroup and suppose that the coset map $q: G \rightarrow G / K$ with $q(g)=K g$ is homotopic to a constant function. Then $K=G$.

With Lemma 1.6, the proof of Theorem 1.1 is easily completed: Under the hypotheses of the theorem, by Lemmas 1.4 and 1.5, the map $f: G \rightarrow x G$ given by $f(g)=x g$ is homotopic to a constant. But this map is equivalent to the quotient map $G \rightarrow G / G_{x}$, where $G_{x}=\{g \in G \mid x g=x\}$ is the isotropy group of $G$ at $x$. By Lemma 1.6 we have $G=G_{x}$, which shows that $x$ is a fixed point.

The proof of Lemma 1.6 was implicitly given in [6] (see 2.9, p. 300 and 
2.7, p. 342). An alternative proof was suggested by Lawson and Madison. We present a slightly more general version which may be of independent interest.

THEOREM 1.7. Let $n \mapsto X_{n}, Y_{n}$ be two functors from a directed set into the category of compact spaces (i.e., two inverse systems) and $f_{n}: X_{n} \rightarrow Y_{n} a$ natural transformation with the induced map $f: X \rightarrow Y$ between the limits $X=$ $\lim X_{n}, Y=\lim Y_{n}$. Assume the following hypotheses:

(i) All $X_{n}$ are manifolds with components $X_{n i}$ of dimension $d(n, i)$, and the homomorphism $H^{d(n, i)}\left(p_{n}\right)$ induced by the limit map $p_{n}: X \rightarrow X_{n}$ does not annihilate the direct summand $H^{d(n, i)}\left(X_{n i}\right)$ of $H^{d(n, i)}\left(X_{n}\right)$, with Cech cohomology relative to some fixed coefficient group.

(ii) All $f_{n}$ are surjective fibrations (i.e., have the homotopy lifting property).

Then all maps in the homotopy class of $f$ are surjective.

Before we prove the theorem, we make a few remarks and draw some conclusions. A space $X$ which is the projective limit of manifolds $X_{n}$ with property (i) has been called a limit manifold (see [6, p. 344]). The main examples for limit manifolds are compact groups [6, p. 302]; however, it is not at all clear that homogeneous spaces of compact groups are limit manifolds.(2) On the other hand, they have all the properties of the space $Y$ in 1.7: Indeed if $G$ is a compact group and $K$ a closed subgroup, let $n$ range through a filter base of compact normal subgroups of $G$ such that $G / n$ is a Lie group. Then the assumptions of Theorem 1.7 are satisfied with $X_{n}=G / n, X=G, Y_{n}=G / K n, Y=G / K, f_{n}: G_{n}$ $\rightarrow G / K n$ the quotient fibration. Therefore we obtain

COROLlARY 1.8. Let $G$ be a compact group, $K$ a closed subgroup and $q: G \rightarrow G / K$ the quotient map. Suppose that $q^{\prime}: G \rightarrow G / K$ is homotopic to $q$. Then $q^{\prime}$ is surjective.

Note that this immediately yields the missing proof of Lemma 1.6. A slightly more general version of 1.8 is a direct consequence:

COROLlaRY 1.9. Let $G$ be a compact group, $K_{1}, K_{2}$ closed subgroups and $r: G / K_{1} \rightarrow G / K_{2}$ the natural map with $r\left(K_{1} g\right)=K_{2} g$. Suppose that $r^{\prime}: G / K_{1}$ $\rightarrow G / K_{2}$ is homotopic to $r$; then $r^{\prime}$ is surjective.

Proof. Let $q_{1}: G \rightarrow G / K_{1}$ be the quotient map and apply 1.8 to $q=$ $r q_{1}, q^{\prime}=r^{\prime} q_{1}$.

With $K_{1}=K_{2}$ this is implicit in Madison [7]. We mention a few further consequences (although they will not be used in the further discussion.) For simplicity we will say that a compact space $Y$ is a quasi-manifold if there is a

(2)There are limit manifolds which are not the homogeneous space of any locally compact group (see [9, Theorem 4, p. 17]). 
family $X_{n}, Y_{n}, f_{n}$ satisfying the hypotheses of Theorem 1.7. Thus any limit manifold is a quasi-manifold, and so is any homogeneous space of a compact group.

COROLLARY 1.10. If a continuous self-map of a quasi-manifold is homotopic to the identity, then it is surjective.

Proof. If $g: Y \rightarrow Y$ is homotopic to $1_{Y}$, then $g f: X \rightarrow Y$ is in the homotopy class of $f$, hence is surjective by 1.7 . Hence $g$ is surjective.

Proposition 1.11. Let $S$ be a compact monoid on a quasi-manifold. Then the arc component $S_{a}$ of the identity is contained in the group of units $H=H(1)$ of $S$. In particular, if the arc component of the identity is dense in $S$, then $S$ is a group.

Proof. If $s \in S_{a}$, then the left translation $L_{s}: S \rightarrow S, L_{s}(x)=s x$, is homotopic to $L_{1}=\mathrm{id}_{s}$. Hence $L_{s}$ is surjective by 1.10 . But then $s \in H(1)$ (see $[6,2.3$, p. 17]).

COROLlary 1.12 (MADISON [7]). Any connected topological monoid on the homogeneous space of a compact group is necessarily a group.

PROOF. The arc component of the identity in a compact group is dense in the identity conponent. Hence in a connected compact homogeneous space of a compact group all arc components are dense. The assertion then follows from 1.11 .

It remains to prove Theorem 1.7.

Proof of THEOREM 1.7. Suppose that $f^{\prime}: X \rightarrow Y$ is a nonsurjective map which is homotopic to $f$. Then there is an index $k$ such that $q_{k} f^{\prime}: X \rightarrow Y_{k}$ is not surjective, where $q_{k}: Y \rightarrow Y_{k}$ denotes the limit map. Since $f_{k}$ is a fibration by (ii), the homotopy $q_{k} f \sim q_{k} f^{\prime}$ lifts, i.e., there is a map $p_{k}^{\prime} \sim p_{k}$ with $q_{k} f^{\prime}=$ $f_{k} p_{k}^{\prime}$. Since this last map is not surjective, but $f_{k}$ is by (ii), then $p_{k}^{\prime}$ fails to be surjective. Hence there must be an index $i$ such that $X_{k i} \nsubseteq p_{k}^{\prime}(X)$. Let $s: X_{k} \rightarrow$ $X_{k i}$ be the retraction which restricts to the identity on $X_{k i}$ and maps the complement to a point. If $d=d(i, n)$, then $H^{d}\left(s p_{k}\right) \neq 0$ by condition (i). By the homotopy axiom we conclude $H^{d}\left(s p_{k}^{\prime}\right) \neq 0$. On the other hand, let $U$ be an open $d$-cell in $X_{i k}$ which does not meet $p_{k}^{\prime}(X)$. Then $s p_{k}^{\prime}$ factors through the inclusion map $j: X_{k i} \backslash U \rightarrow X_{k i}$. But $H^{d}(j)=0$ (as is well known and follows from the exact cohomology sequence and the fact that $H^{n}(\bar{U}, \bar{U} \backslash U) \cong H^{n}(X, X \backslash U) \rightarrow$ $H^{n}(X)$ is an isomorphism). Hence $H^{d}\left(s p_{k}^{\prime}\right)=0$, and this is a contradiction.

2. Some results about transformation semigroups. Let $T$ be an $I$-semigroup, i.e., a topological monoid on a compact connected totally ordered space such that 
0 and 1 are endpoints. Assume that $T$ acts (on the right) continuously on the compact space $X$ with $x 1=x$ and $x(s t)=(x s) t$ for all $x \in X, s, t \in T$. Let $A$ be an open proper subset of $X$ which is invariant under $T$, i.e., satisfies $A T \subseteq A$ (and hence $A T=A$ ). Let $B$ be its boundary and $C$ its (closed) complement.

Definition 2.1. We say that $A$ is transversal for $T$ if

(0) $X 0 \subseteq A$.

(t) $b t \in B$ with $b \in B, t \in T$, implies $b t=b$.

We remark that condition (0) immediately implies that every orbit $c T, c \in$ $C$, meets $B$, since it is connected and contains $c 0 \in X 0 \subseteq A$. Condition (t) says that each orbit $c T, c \in C$, meets $B$ in exactly one point, for if $c s, c t \in B$ with $s \leqslant t$, then we write $s=t u$ with some $u \in T$ and set $c t=b \in B$, so that $c s=c t u=b u \in B$; hence by condition ( $\mathrm{t})$ we have $b u=b$, and so $c s=c t$.

The significance of transversality for our purposes lies in the following result:

PROPOSITION 2.2. Let the I-semigroup $T$ act (on the right) on a space $X$. If $A$ is a proper open subset of $X$ which is invariant and transversal for $T$, and if its boundary $B$ is compact, then $B$ is a retract of the complement $C=X \backslash A$.

Proof. For each $c \in C$ we let $f(c) \in T$ be given by $f(c)=\min \{t \in T \mid$ $c t \in C\}$. Now $t<f(c)$ implies $c t \in A$, so $c f(c) \in \bar{A} \cap C=B$. Hence $c f(c)$ is the unique element in $c T \cap B$. We define $r: C \rightarrow B$ by $r(c)=c f(c)$. Then $r(b)=b$ for $b \in B$ and $r$ is a retraction. We show its continuity: Let $c_{k}$ be a net in $C$ converging to $c$. Let $c_{k(j)}$ be an arbitrary subnet such that $b=\lim r\left(c_{k(j)}\right)$ exists in $B$. Let $c_{i}^{\prime}$ be a subnet of $c_{k(j)}$ such that $\lim f\left(c_{i}^{\prime}\right)=t$ exists in $T$. Then $b$ $=\lim r\left(c_{i}^{\prime}\right)=\lim c_{i}^{\prime} f\left(c_{i}^{\prime}\right)=c t \in c T \cap B$. But the unique element in $c T \cap B$ is $r(c)$. Hence $b=r(c)$. Since all cluster points of the net $r\left(c_{k}\right)$ agree with $r(c)$ and $B$ is compact, we have $\lim r\left(c_{k}\right)=r(c)$, which shows the continuity of $r$.

There is a slightly better result, which we do not need but which we can now obtain rather quickly:

COROLlaRY 2.3. Under the hypotheses of 2.2, the boundary $B$ is a deformation retract of $C$ in the sense that there is a continuous function $F: C \times T$ $\rightarrow C$ such that $F(c, 1)=c, F(c, 0) \in B$, and $F(b, t)=b$ for all $c \in C, b \in B$, and $t \in T$.

Proof. We define $F(c, t)=c(\max \{t, f(c)\})$ with $f(c)$ as in the proof of 2.2. Then $F(c, 1)=c 1=c, F(c, 0)=c f(c)=r(c) \in B, F(b, t) \in b T \cap B$; hence $F(b, t)=b$. There remains the continuity: Let $\left(c_{k}, t_{k}\right)_{k \in K}$ be an arbitrary net on $C \times T$ with limit $(c, t)$. Let $K_{1}=\left\{k \in K \mid t_{k} \leqslant f\left(c_{k}\right)\right\}, K_{2}=$ 
$\left\{k \in K \mid f\left(c_{k}\right) \leqslant t_{k}\right\}$. If $K_{1}$ is cofinal we have

$$
\begin{aligned}
\lim _{k \in K_{1}} F\left(c_{k}, t_{k}\right) & =\lim _{k \in K_{1}} c_{k} f\left(c_{k}\right)=\lim _{k \in K_{1}} r\left(c_{k}\right)=r(c) \\
& =c f(c)=c(\max \{t, f(c)\})=F(c, t),
\end{aligned}
$$

by the continuity of $r$ in 2.2 and the definition. If $K_{2}$ is cofinal, then

$$
\begin{aligned}
\lim _{k \in K_{2}} F\left(c_{k}, t_{k}\right) & =\lim _{K \in K_{2}} c_{k} t_{k}=c t \\
& =c(\max \{t, f(c)\})=F(c, t)
\end{aligned}
$$

by the continuity of the action. Thus we have $\lim F\left(c_{k}, t_{k}\right)=F(c, t)$; this shows the continuity of $F$.

We do not have a very good idea how frequent transversal invariant sets are; we will obtain them in the case of monoids in which a D-class separates the space:

Definition 2.4. Let $S$ be a compact connected semigroup. For a $D$-class $D$ which is different from the minimal ideal $M(S)$, let $I(D)$ denote the maximal ideal of $S$ not meeting $D$ (see $[6,3.2$, p. 28]). We say $D$ separates if $S W$ is disconnected and $I(D)$ is a component of $S \backslash D$.

Note that $H(1)=D(1)$ never separates since $S \backslash H(1)=I(D(1))$.

Suppose $D$ is a separating $D$-class of the compact connected semigroup $S$, and let $\Delta$ be the boundary of $I(D)$. Then $D \subseteq \Delta$ (see $[6,3.2$, p. 28]). Moreover, if $s \in S \backslash(D \cup I(D))$, then since $I(D)$ is a component of $S \backslash D$, there is an open set $U \subseteq S$ with $s \in U$ and $(U \cap S \backslash D) \cap I(D)=\square$. But, $D$ is closed, so $U \cap S \backslash D$ is an open subset of $S$ which contains $s$ and misses $I(D)$. Therefore $s \notin \Delta$. Since $I(D)$ is open, this shows $\Delta=D$.

LEMMA 2.5. Let $S$ be a compact connected semigroup with a right identity 1 and with an I-semigroup $T$ from 1 to the minimal ideal. Let $T$ act on $S$ on the right under multiplication. Then any right ideal $I$ is $T$-invariant. If $D$ is a separating D-class other than $M(S)$ then $I(D)$ is transversal for $T$ if at least one of the following two hypotheses is satisfied:

(a) 1 is an identity and is the only idempotent in or above $D$ (in the D. quasi-order).

(b) $D$ is an L-class.

Proof. Let $D$ be a separating $D$-class other than $M(S)$. If $e$ is the zero of $T$, then $e \in M(S)$ by hypothesis and so $S e \subseteq M(S) \subseteq I(D)$.

The transversality: Let $d \in D$ and suppose $d t \in D$ for some $t \in T$. Since $D$ is the boundary of $I(D)$ we have to show that $d t=d$. In the case of hypothesis (a), the relation $d t \in D$ implies the existence of two units $g, h \in H(1)$ such that $g d t h=d\left[6\right.$, Proposition 4.18, p. 38]. By induction it follows that $g^{n} d(t h)^{n}$ $=d$. Then there is a net $n(k)$ of natural numbers such that $\lim \left(g^{n(k)},(t h)^{n(k)}\right)=$ 
$(e, f)$ exists and $e, f \in E(S)$ [6, Proposition 1.18, p. 15]. So $e d f=d$; in particular $d f=d$. Thus $f$ is an idempotent which is above $d$ in the L-quasi-order. Hence $f=1$. But $\lim (t h)^{n(k)}=1$ implies $t=1$ (see, e.g., [6, Lemma 2.5, p. 18]). Hence $d t=d 1=d$, as was to be shown.

In the case of hypothesis (b) we have $d t \in L(d)$. Thus adt $=d$ for some $a \in S$. Again we obtain $a^{n} d t^{n}=d$, and thus $e d f=d$ with idempotents $e$ and $f$ (where $f=\lim t^{n}$, since $T$ is an $I$-semigroup). But we have also $b d=d t$ for some $b \in S$; since $t f=f$, we may conclude $d=d f=d t f=b d f=b d=d t$, as was to be shown.

Under the hypotheses of Lemma 2.5 the $I$-semigroup $T$ acts on the right on $S / L$ via $L(s) t=L(s t)$, since $L$ is a right congruence. Let $D / L$ and $I(D) / L$ be the images of $D$ and $I(D)$ in $S / L$. Then we have

LEMma 2.6. Under the hypotheses of Lemma 2.5 the set $I(D) / L$ is an invariant transversal set for $T$.

Proof. Let $L(d) t \in D / L$ for $d \in D$. Then $d t \in D$. By 2.5 this means $d t$ $=d$; hence $L(d) t=L(d)$.

We may collect our information in the following

Proposition 2.7. Let $S$ be a compact connected semigroup with right identity 1. Suppose that

(i) $S$ contains an I-semigroup from 1 to $M(S)$.

(ii) $S$ contains a separating D-class $D=M(S)$ such that one of the two hypotheses holds:

(a) The only idempotent in or Dabove $D$ is 1 , and 1 is an identity.

(b) $D$ is an L-class.

Then $D$ is a (deformation) retract of $S \bigvee(D)$ and $D / L$ is a (deformation) retract of $(S / L) \backslash(I(D) / L)$.

Proof. This follows from 2.2 (resp. 2.3, 2.5, 2.6).

From this result and Theorem 1.1 we derive, in particular, the following technical, but for certain applications, crucial conclusion.

PROPOSITION 2.8. Let $S$ be a compact connected semigroup and $D \neq$ $M(S)$ a D-class of $S$ without idempotents. Let e be a minimal element of $\{f \in$ $E(S): f S f \cap D \neq \square\}$, and let $d \in D$ with $d \leqslant e(H)$. If $D \cap$ eSe separates eSe (in the sense of 2.4), then $d H(e) \subseteq H(e) d \subseteq L(d)$.

REMARK. Since $e S e$ is a retract of $S$, the monoid $e S e$ is compact and connected, and the set $D \cap e S e$ is one of its $D$-classes by $[6,3.16$, p. 32]. 
Proof. Let $D^{\prime}=D \cap e S e$, and suppose $f \in E(e S e)$. If $f S f \cap D^{\prime}=\square$, then $f S f$ is connected and meets $M(e S e)$, so $f S f \subseteq I\left(D^{\prime}\right)$ since $I\left(D^{\prime}\right)$ is a component of $e S e D^{\prime}$. Let $T_{1}$ be a solenoidal semigroup containing $e$ as identity but not contained in $H(e)$. If $f$ is the idempotent in $M\left(T_{1}\right)$, then $f \neq e$, and so $f S f \cap$ $D^{\prime}=\square$ since $e$ is minimal in this respect by hypothesis. Thus $T_{1} \cap D^{\prime} \neq \square$, since $T_{1} \cup f S f$ is a connected subset of $e S e D^{\prime}$ which meets $I\left(D^{\prime}\right)$ otherwise. Thus $f$ must be $H$-below some element of $D^{\prime}$. Consider $\widetilde{S}=e S e /(e S f S e)$, the image $\widetilde{D}$ of $D \cap e S e$ in $\widetilde{S}$ and the image $\widetilde{T}$ of $T_{1}$ in $\widetilde{S}$. Then $\widetilde{D}$ separates $\widetilde{S}$, since $D \cap$ $e S e$ separates $e S e$, and the identity $\widetilde{e}$ is the only idempotent of $\widetilde{S}$ above $\widetilde{D}$. Moreover, $\widetilde{T}$ is an $I$-semigroup from $\widetilde{e}$ to zero in $\widetilde{S}$. Hence, by $2.7, \widetilde{D} / \widetilde{L}$ is a retract of $(\widetilde{S} / \widetilde{L}) \backslash(\widetilde{I}(\widetilde{D}) \widetilde{L})$. The Rees quotient map eSe $\rightarrow \widetilde{S}$, however, maps $e \dot{S} e \backslash I(D \cap e S e)$ homeomorphically onto $\widetilde{S} \backslash I(\widetilde{D})$, since it only collapses the ideal $e S f S e \subseteq I(D \cap e S e)$. Hence $(D \cap e S e) / L_{e S e}$ is a retract of $X=(e S e \backslash I(D \cap e S e)) / L_{e S e}$. But $D \cap e S e$ $=H(e) d H(e)$ and $L_{e S e}\left(d^{\prime}\right)=H(e) d^{\prime}$ for all $d^{\prime} \in D \cap e S e$ [6, Proposition 4.18, p. 38].

Hence, if we let the compact group $G=H(e)$ operate on $X$ on the right by $L_{e S e}(s) h=L_{e S e}(s h)$ then $(D \cap e S e) / L_{e S e}$ is the orbit $x G, x=H(e) d=L_{e S e}(d)$. Moreover, $x_{0}=L_{e S e}(e)=H(e)$ is a fixed point for the action of $G$, and the orbits $x G$ and $\left\{x_{0}\right\}$ are in the same arc component, since $T_{1}$ contains $e$ and meets $H(e) d H(e)=D \cap e S e$. Thus Theorem 1.1 applies and shows that $x$ is a fixed point. Hence $x G=\{x\}$, and thus $H(e) d H(e) \subseteq H(e) d$. Hence $d H(e) \subseteq H(e) d=$ $L_{e S e}(d) \subseteq L_{S}(d)$.

We have an addition to Proposition 2.7 which is proved independently of the methods developed in this section but which nevertheless will be useful for the same type of application of the transformation group theoretical methods which we have seen in Proposition 2.8. First, we make a definition:

DEFINITION 2.9. We call an ideal $P$ in a topological semigroup $S$ a prime ideal iff it is open and if $S \backslash P$ is a subsemigroup. A J-class $J$ is called a prime generator iff $I(J)$ (the maximal ideal not containing $J$ ) is prime.

EXAMPLE. If $S$ is a compact monoid then $S W(1)$ is a prime ideal, and $H(1)$ is a prime generator. If $S$ is an $I$-semigroup, then the prime ideals are precisely the open ideals with idempotent suprema, and this maintains for all hormoi.

LEMMA 2.10. Let $S$ be a compact topological semigroup and suppose that the $D$-class $D$ is a prime generator. Then $D$ is a retract of $S \backslash I(D)$ and $D / L$ is a retract of $(S / L) \backslash(I(D) / L)$.

Proof. By definition $S^{\prime}=S \backslash(D)$ is a semigroup. If $s^{\prime} \in S^{\prime}$, then $S^{1} s^{\prime} S^{1}$ $\cap D \neq \square$, where $S^{1} s S^{1}=S s S \cup S s \cup s S \cup\{s\}$, because otherwise $s^{\prime} \in S^{1} s^{\prime} S^{1} \subseteq$ $I(D)$, which is impossible. But each ideal is $D$-saturated; hence $D \subseteq S^{1} s^{\prime} S^{1}$. 
Hence $D \leqslant D^{\prime}$ in $S$ for all $D$-classes $D^{\prime}$ of $S$ contained in $S^{\prime}$. But $I(D)$, hence $S^{\prime}$, is $D$-saturated; thus $D \leqslant D^{\prime}$ holds in $S^{\prime}$ relative to the $D$-quasi-order in $S^{\prime}$. Hence $D$ is the minimal ideal of $S^{\prime}$. It is well known that for any idempotent $e \in D$ the function $r: S^{\prime} \rightarrow D$ with $r(s)=s(e s e)^{-1} s$ is a continuous retraction, where (ese $)^{-1}$ is the inverse of ese in the group $e S^{\prime} e=H(e)$ (see, e.g., [2, Theorem 3.5, iii.b, p. 67]).

3. Applications to the centralizing conjecture. If $S$ is a monoid and $X \subseteq$ $S$ we write $Z(X, S)$ for the centralizer $\{s \in S \mid x s=s x$ for all $x \in X\}$ of $X$ in $S$, and $Z(X, S)_{0}$ denotes the component of 1 in $Z(X, S)$. The following is an inessentially modified version of the conjecture described in the introduction:

CONJECTURE 3.0. In any compact connected monoid $S$ one has $Z(H(1), S)_{0}$ $\cap M(S) \neq \varnothing$.

This section discusses some partial results confirming the conjecture. We will base all of the following results on the discussions of $\S \S 1$ and 2 and the following theorem, due to the authors [5].

THEOREM 3.1. If $S$ is a compact connected monoid and $G \subseteq H(1)$ a group of units such that $s G \subseteq G$ s for all $s \in S$ (i.e., that $G$ is left normal), then $Z(G, S)_{0}$ $\cap M(S) \neq \varnothing$.

We begin the section with a lemma:

LEMMA 3.2. Let $S$ be a compact connected semigroup and e an idempotent outside the minimal ideal such that $e$ is isolated in $E(e S e)$. Then the following two conditions are equivalent:

(A) $Z(H(e), e S e)_{0} \nsubseteq H(e)$.

(B) If $U$ is any open set of $S$ containing $H(e)$ then there is an element $x \in$ $(U \cap e S e) \backslash H(e)$ with $x H(e) \subseteq L(x)$ (where $L(x)=L_{S}(x)$ is the L-class of $x$ in $S$ ).

Proof. (A) trivially implies (B), for if (A) is satisfied and $U$ is an open neighborhood of $H(e)$, then we find an element $x$ in $\left(U \cap Z(H(e), e S e)_{0}\right) \backslash H(e)$, and then $x H(e)=H(e) x \subseteq L(x)$.

Now suppose $(B)$. Let $X=\{x \in e S e \mid x H(e) \subseteq L(x)\}$; since $x H(e) \subseteq e S e$ and $L_{S}(x) \cap e S e=L_{e S e}(x)\left[6,3.16\right.$, p. 32], we have $x \in X$ iff $x H(e) \subseteq L_{e S e}(x)$. From the fact that $e$ is isolated in $E(e S e)$ we conclude that there is an open neighborhood $V$ of $H(e)$ in $e S e$ such that $v \in V$ implies $L_{e S e}(v)=H(e) v[6,4.20$, p. 39]. Let $N$ be the left normalizer $\{x \in e S e \mid x H(e) \subseteq H(e) x\}$ of $H(e)$ in eSe (see $[5,2.1]$ ). We have $X \cap V \subseteq N$. Since every neighborhood of $H(e)$ in eSe contains elements of $X$, then $H(e)$ is not open in $N$. Thus the identity component 
$N_{0}$ of $N$ is not contained in $H(e)$ by the one parameter semigroup theorem [6, Theorem III, p. 117]; let $s \in N_{0} \backslash H(e)$. Then $I=e S e s e S e$ is an ideal in eSe which does not meet $H(e)$; since $S$ is connected, $e S e$, and hence $I$, are connected; since $I h=I$ for each $h \in H(e)$, then $N_{0} h$ meets $I$ for each $h \in H(e)$ and thus $T=$ $N_{0} H(e) \cup I$ is a compact connected space. Since $N_{0}$ is invariant under the inner automorphisms of $N$, then $N_{0} h=h N_{0}$ for all $h \in H(e)$ and thus $T$ is a semigroup. Then $T / I$ is a compact semigroup with zero in which the image of $H(e)$ is left normal. By Theorem 3.1, its centralizer in $T / I$ is connected. Hence $Z(H(e), T)$ $U I$ is connected, from which we conclude that $Z(H(e), T)_{0} \cap I \neq \varnothing$. Since $Z(H(e), T)_{0} \subseteq Z(H(e), e S e)_{0}$, then certainly $Z(H(e), e S e)_{0} \nsubseteq H(e)$.

In the following theorem we give a list of diverse properties of a monoid each of which implies condition (B) of 3.2, hence (A) of 3.2.

THEOREM 3.3. Let $S$ be a compact connected semigroup and e an idempotent outside the minimal ideal which is isolated in $E(e S e)$. Then we have

$$
Z(H(e), e S e)_{0} \nsubseteq H(e)
$$

provided at least one of the following conditions. is satisfied:

(1) There is a closed congruence $Q$ on $S$ such that $H(e) \subseteq Q(e)$ and $Q \cap R$ $\subseteq L$

(2) H is a congruence.

(3) $L$ is a congruence.

(4) D is a congruence.

(5) $x S \subseteq S x$ for all $x \in S$.

(6) The H-quasi-order is total.

(7) The L-quasi-order is total.

(8) The D-quasi-order is total.

Proof. (1) $\rightarrow$ (B). Let $x \in e S e$; then $x H(e) \subseteq Q(x) Q(e) \subseteq Q(x e)=Q(x)$. But also $x H(e) \subseteq R(x)$; thus $x H(e) \subseteq(Q \cap R)(x) \subseteq L(x)$.

(2) $\rightarrow$ (1). Take $Q=H$.

(3) $\rightarrow$ (1). Take $Q=L$.

$(4) \rightarrow(\mathrm{B})$. Since (B) of 3.2 is a local property of eSe around $H(e)$, there is no loss of generality in assuming that $e S e$ has a zero 0 and no other idempotents outside $e$ (if this is not the case we form the Rees quotient of $S$ modulo the closed ideal $S(E(e S e) \backslash\{e\}) S)$. Then there is a one parameter semigroup $T$ from $e$ to $0[6$, Theorem III, p. 117].

Since $D$ is a congruence, then $D(T)=\bigcup\{D(t) \mid t \in T\}$ is a closed subsemigroup, and since $D_{S}(t) \cap e S e=D_{e S e}(t)\left[6,3.16\right.$, p. 32], then $\widetilde{T}=D_{S}(T) \cap e S e$ $=D_{e S e}(T)=\bigcup\left\{D_{e S e}(t) \mid t \in T\right\}$ is a closed subsemigroup. 
For each $t \in T, D_{e S e}(t)=H(e) t H(e)$ since $E(e S e)=\{e, 0\}$, and so the same is true for each $t \in \widetilde{T}$. It follows that $D_{\widetilde{T}}=D_{\widetilde{T}}$ is a congruence on $\widetilde{T}$ and that $T$ is a cross section for $D_{\widetilde{T}}$, whence $\widetilde{T} / D_{\widetilde{T}}$ is an arc. If $D_{\widetilde{T}}(t)$ is a $D$-class of $\widetilde{T}$ other than $H(e)$ or 0 , then $D_{\widetilde{T}}(t)$ separates $T / D_{\widetilde{T}}$ into two components, one of which is the image of $I\left(D_{\widetilde{T}}(t)\right)$. It follows that $D_{\widetilde{T}}(t)$ separates in the sense of 2.4, so Proposition 2.8 applies to $\widetilde{T}$, showing $t H(e) \subseteq H(e) t \subseteq L(t)$ for all $t \in \widetilde{T}$. This implies (B) of 3.2.

$(5) \rightarrow(3)$. See $[6,3.19$, p. 33]

$(6) \rightarrow(7) \rightarrow(8)$. Clear.

$(8) \rightarrow(B)$. If the $D$-quasi-order on $S$ is total, then the $D$-quasi-order on $e S e$ is total. Again, without loss of generality we assume that $E(e S e)=\{0, e\}$. Since the $D$-quasi-order is total, then every $D$-class $D_{\text {ese }}(e s e)$ with ese $\notin\{0\} \cup$ $H(e)$ separates $e S e$. Proposition 2.8 then shows that $d H(e) \subseteq H(e) d \subseteq L(d)$ for all $d \in e$ Se.

This theorem has several interesting consequences. The first one is a result in [6], which up to this point remained unproved, since the proof given in [6] contained a gap on p. 342 (proof of 2.6) which was discovered by J. H. Carruth in 1971 and which could not be repaired in order to salvage the proof of Theorem VI (p. 177) in [6]. First a definition:

Definition 3.4. If $S$ is a compact monoid we say that the D-quasi-order is total near 1 if there is a $D$-class $D$ satisfying the following two conditions:

(i) $D=M(S)$ or $D$ is separating (see 2.4 );

(ii) the interval $[D, H(1)]=\left\{D^{\prime} \in S / D \mid D \leqslant_{0} D^{\prime} \leqslant_{0} H(1)\right\}$ is a totally ordered subspace and a neighborhood of $H(1)$.

THEOREM 3.5. Let $S$ be a compact connected monoid in which $H(1)$ is not open and in which 1 is isolated in $E(S)$. If the D-quasi-order is total near $H(1)$, then there is a one parameter semigroup $f: H \rightarrow S$ such that

(i) $f(0)=1$.

(ii) $f(\mathbf{H}) \nsubseteq H(1)$.

(iii) $f(\mathrm{H}) \subseteq Z(H(1), S)$.

In particular, there is a cylindrical semigroup $C=\overline{f(\mathbf{H})} H(1) \subseteq S$ which is a neighborhood of 1. If $E(S) \subseteq\{1\} \cup M(S)$ and the D-quasi-order is total, then $S=$ $C \cup M(S)$.

Proof. By hypothesis there is a closed ideal $I=S D S$ of $S$ such that $S / I$ is totally $D$-quasi-ordered and that card $E(S / I)=2$. Then the hypothesis (8) of Theorem 3.3 is satisfied; hence, by 3.3, $Z=Z\left(H_{S / I}(1), S / n_{0} \nsubseteq H_{S / I}(1)\right.$. By the one parameter semigroup theorem [6], $Z$ contains a nondegenerate one parameter semigroup which is not contained in $H_{S / I}(1)$. Since $S \rightarrow S / I$ is a 
local isomorphism on $S \backslash$, we find $f$ with properties (i), (ii), (iii). Then $f(r) h=$

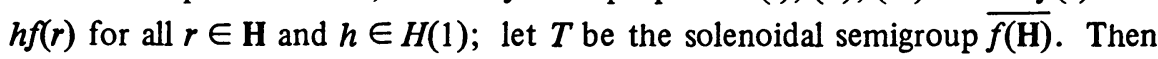
the map $T \times H(1) \rightarrow S$ given by $(t, h) \mapsto t h$ is a continuous morphism with image $C$.

Assume for the moment that $E(S)=\{1,0\}$. Then all $D$-classes $D(s) \neq\{0\}$ are of the form $D(s)=H(1) s H(1)\left[6,4.20\right.$, p. 39]. In particular, $S=\bigcup\left\{T_{s} \mid\right.$ $s \in S\}$ is connected, and $E(S) \subseteq T$. Hence $C$ is $D$-saturated and $D_{S}$ induces $D_{C}$ on $C$. The cylindrical semigroup $C$ is totally $D$-quasi-ordered and satisfies $D=$ $H$. Now the partially ordered space $S / D$ is an interval with minimum $\{0\}$ and maximum $H(1)$ relative to $\leqslant_{D}$ and the order is total near the maximum, i.e., there is a $D \in S / D$ such that the interval $[D, H(1)]$ is a totally ordered neighborhood of $H(1)$ and that $D=M(S)=\{0\}$ or $D$ separates. But $C / D$ is a totally ordered compact connected subset containing $\{0\}$ and $H(1)$; hence $D \in C / D$ since $D=$ $\{0\}$ or $D$ is separating.

Now $\left\{D^{\prime} \in C / D \mid D \leqslant D^{\prime}\right\}$ is a connected subset of the totally ordered set $[D, H(1)]$ containing $D$ and $H(1)$, and must therefore agree with $[D, H(1)]$. Hence $[D, H(1)] \subseteq C / D$, i.e., $C$ is a neighborhood of 1 .

If the $D$-quasi-order on $S$ is total, we may take $D=\{0\}$ and conclude that $S / D=[D, H(1)] \subseteq C / D$, i.e., $S \subseteq C \subseteq S$.

If we now drop the hypothesis that $E(S)=\{1,0\}$, then we apply the preceding observation to $S / I$ where we have $E(S / I)=\{\{I\}, 1\}$. By what we have shown, $(C \cup I) / I$ is a neighborhood of 1 in $S / I$; hence $C \cup I$ is a neighborhood of 1. Since $I$ is closed and $1 \notin I$, then $C$ is a neighborhood of 1 . If $\leqslant_{0}$ is total and $E(S) \subseteq\{1\} \cup M(S)$, then we may take $D=M(S)$; we have seen $S / I=(C \cup I) / I$; hence $S=C \cup I=C \cup M(S)$.

Recall again that a cylindrical semigroup is the quotient of a direct product of a solenoidal compact monoid and a compact group; the structure of cylindrical monoids is thoroughly understood in great detail (see [6, Chapter B, §2]).

In the light of this fact the following corollary, which still carries all of the essential information of the preceding theorem, is somewhat easier to recall:

COROLlaRY 3.6. Let $S$ be a compact monoid with $E(S)=\{0,1\}$. Then the following statements are equivalent;

(1) $S$ is cylindrical.

(2) D-quasi-order is total and H(1) is not open.

The next centralizing result follows rather directly from Theorem 3.3.

Proposition 3.7. Suppose that $S$ is a compact connected monoid satisfying at least one of the hypotheses (2)-(8) of 3.3. 
Let $G \subseteq H(1)$ be a closed group of units, and assume in addition that the following hypothesis is satisfied:

(Z) If $e \in E(S)$ is not isolated in $E(e S e)$, and e commutes with $G$, i.e., $e G$ $=G e$, then $e$ is not isolated in $E(e Z(G, S) e)$.

Then $Z(G, S)_{0} \cap M(S) \neq \varnothing$.

The hypothesis $(\mathrm{Z})$ is implied by the following:

$\left(\mathrm{Z}^{\prime}\right)$ if $e \in E(S)$ is not isolated in $E(e S e)$, and e commutes with $H(1)$, then $e$ is not isolated in $E(Z(H(e), e S e))$.

Proof. First we show that (Z') implies (Z). Since $Z(H(1), S) \subseteq Z(G, S)$, it is sufficient to observe that $Z(H(e), e S e) \subseteq Z(H(1), S)$. Indeed, if $z \in$ $Z(H(e), e S e)$ and $e H(1)=H(1) e$, then for each $h \in H(1)$ we have $z h=(z e) h=$ $z(e h)=z(e h e)=(e h e) z$ since $h e=e h e=e h \in H(e) ;$ but $(e h e) z=(h e) z=h z$.

Now we show that $(Z)$ implies the centralizing conjecture for $G$. We apply the chaining theorem $[6$, Theorem IV, p. 132] to $Z=Z(G, S)$. Take an idempotent $e \in Z$ with $e \notin M(S)$. If $e$ is isolated in $E(e Z e)$, then $e$ is isolated in $E(e S e)$ by $(\mathrm{Z})$; then, by Theorem 3.3, we have $Z(H(e), e S e)_{0} \nsubseteq H(e)$. Thus $H_{Z}(e)=Z \cap H(e)$ is not open in eZe. This is the hypothesis required in the chaining theorem, which then shows that $Z_{0} \cap M(Z) \neq 0$. We must show that $M(Z) \subseteq M(S)$. Let $e=e^{2} \in M(Z)$. By $(\mathrm{Z})$ and since $\{e\}=E(e Z e)$ we know that $e$ is isolated in $E(e S e)$. If we had $e \notin M(S)$, then Theorem 3.3 would apply and show that there would exist elements of $Z(H(e), e S e) \subseteq Z(H(1), S) \subseteq Z$ below $e$ in the H-order, contradicting the fact that $e \in M(Z)$.

If Green's relation $H$ is a congruence, then for any idempotent $e \in S$ we have $e H(1) \subseteq H(e) H(1) \subseteq H(e 1)=H(e)$, similarly, $H(1) e \subseteq H(e)$. Hence $e$ commutes with $H(1)$ because of $e h=e h e=h e$. The preceding proposition then immediately yields the following result conjectured by J. H. Carruth:

THEOREM 3.8. Any compact connected monoid in which Green's relation $H$ is a congruence verifies the centralizing conjecture, i.e., the identity component of the centralizer of the group of units meets the minimal ideal.

Our knowledge of the analogous theorem for Green's relation $L$ or $D$ is incomplete. Some partial results nevertheless can be formulated in this case. Recall that a semigroup $S$ is normal if $S x=x S$ for all $x \in S$. One checks readily that in a normal compact semigroup Green's relations $H, L, R, D$ all collapse and form a congruence (see $[6,3.18(3)$, p. 32$])$. Hence

COROLLARY 3.9. The centralizing conjecture holds in all normal compact connected monoids. 
We point out that the proof of this fact as a portion of $[6$, p. 181, Proposition 9] contains a gap; if $H$ is a congruence on $S$, and if $T$ is an $H$-saturated submonoid of $S$, then the $H$ relation $H_{T}$ of $T$ may be properly smaller than the relation $H \cap(T \times T)$ induced by $H$. In fact, it is not known whether $H_{T}$ is a congruence even in special cases. This difficulty has been overlooked in previous discussions in this context. We remind the reader that, of course, in view of Proposition 3.7 we can still assert that the centralizing conjecture holds if $L$ or $D$ is a congruence and if, in addition, all idempotents $e$ outside the minimal ideal are isolated in $E(e S e)$.

Some of the pathologies which we may expect even in the case that $L$ is a congruence will appear in our discussions of some examples in $\$ 5$ below.

4. The $H \times H$ theorem. This section is concerned with the following issue: Let $S$ be a compact monoid, and let $H=H(1)$ be its group of units. Then the group $H \times H$ acts on $S$ via $s \circ(g, h)=g^{-1}$ sh. Let $Y=S /(H \times H)$ be the orbit space. If $S$ is connected, then so is $Y$.

The orbit space $X$ of the subgroup $H \times\{1\}$ is the space $S / H$ of orbits $H s$, $s \in H$, and $H$ operates on $X$ on the right under $(H s) h=H s h$. There is then a commuting diagram of orbit maps

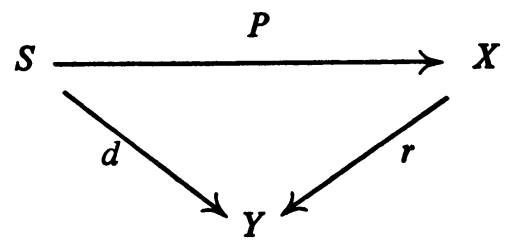

$d(s)=H_{s} H, p(s)=H s, r(H s)=H s H$. We will discuss the case that $Y$ is a totally ordered compact connected space. The principal result of this section will state that, under suitable additional hypotheses, $S$ contains an $I$-semigroup $T$ which commutes elementwise with $H$ such that $S=H T$.

DEFINITION 4.1. We say that $S$ is a TO-monoid iff $S$ is a compact monoid such that the following conditions are satisfied:

(i) $Y=S /(H \times H)$ is a totally ordered space which is compact and connected in its order topology.

(ii) $M(S)=H e H$ for some $e \in E(M(S))$ and $M(S)=\min Y$.

LEMMA 4.2. If $S$ is a TO-monoid, then $S / M(S)$ is connected. If I is a left ideal of $S$ then $(I \cup M(S)) / M(S)$ is connected.

Proof. For each $s \in S$ we have $H s H \subseteq D(S)$, so $S / D$ is a quotient space of $Y$, and is therefore connected. We show that this implies $H(e)$ is not open in $e S e$ for each idempotent $e \in S W(S)$. To the contrary, suppose $e=e^{2} \in S M(S)$ 
and $H(e)$ is open in eSe. Suppose $I(D(e))^{-} \cap D(e) \neq \square$. If $x \in I(D(e)) \cap D(e)$, then there are $a, b \in S$ with $a x b=e$. Since $a I(D(e)) b \subseteq I(D(e))$, we have $e \in$ $I(D(e))^{-}$, and so $e \in e I(D(e))^{-} e \subseteq(e I(D(e)) e)^{-}$. Since $e I(D(e)) e \subseteq e S e$ and $H(e)$ is open in $e S e$, we must then have $e I(D(e)) e \cap H(e) \neq \square$. Since $e I(D(e)) e \subseteq$ $I(D(e))$, we then have $I(D(e)) \cap H(e) \neq \square$. This contradicts the fact that $I(D(e))$ $\cap D(e)=\square$, so we conclude $I(D(e))^{-} \cap D(e)=\square$. This then shows that $I(D(e))=I(D(e))^{-}$, since $I(D(e))$ is the largest ideal of $S$ not meeting $D(e)$. Since $I(D(e))$ is open, $I(D(e))$ is a compact open subset of $S$. Being an ideal, $I(D(e))$ is $D$-saturated, so $I(D(e)) / D$ is open and closed in $S / D$, contradicting the fact that $S / D$ is connected.

Thus, $H(e)$ is not open in eSe for each $e=e^{2} \in S \backslash M(S)$. By the chaining theorem [6, Theorem IV, p. 132], there exists a compact connected submonoid $T \subseteq S$ with $1 \in T$ and $T \cap M(S) \neq \varnothing$. Thus $S=\bigcup\{T s \mid s \in S\}$ and the connected spaces $T s$ all meet $M(S)$. Hence $S / M(S)$ is a union of connected spaces all of which contain the point $M(S)$. Hence $S / M(S)$ is connected. If $I$ is a left ideal then $I=\bigcup\{T s \mid s \in I\}$. By the same argument as before, $(I \cup M(S)) / M(S)$ is connected.

LEMma 4.3. In a TO-monoid we have $H s H=D(s)$ for all $s$.

Proof. If $s \in M(S)$, then $H s H=M(S)$ by 4.1 (ii); hence $H s H=D(s)$. Now let $s \notin M(S)$. Then $I(s)=I(H s H)$, the largest ideal not containing $s$ is not empty $([6,3.2$, p. 28$]$, see also $\S 2$ above) and connected modulo $M(S)$ by 4.2 , since $M(S) \subseteq I(s)$. Since $I(s)$ is $H \times H$ invariant, the image $d(I(S))$ in $Y$ is open, connected, hence an open interval $[M(S), y[$. The boundary of $I(s)$ goes to the boundary $\{y\}$ of $d(I(S))$ in $Y$. Thus $H s H=d(H s H) \in\{y\}$; hence $H s H=y$. Thus $H s H$ is the boundary of $I(s)$ which also contains $D(s)$. Hence $H s H=D(s)$, as asserted.

LEMMA 4.4. If $S$ is a TO-monoid, then the given total order on $S /(H \times H)$ is the D-order. In particular, $H(1)$ is the maximal coset of $S /(H \times H)$.

Proof. Suppose that $s<_{D} t$, i.e., $s \in S t S \backslash D(t)$. Since $S t S \backslash D(t)$ is an ideal not containing $t$, we have $s \in I(t)$. By the proof of Lemma 4.3 we obtain $d\left(I(t)^{-}\right)=[M(S), H t H]$ and $d(I(t))=[M(S), H t H[$, and so $H s H \in d(I(t))$ implies $M(S) \leqslant H s H<H t H$ in the given order of $Y$. Conversely, suppose that $H s H \leqslant$ $H t H$ in the given order of $Y$. The ideal $S t S$ is connected modulo $M(S)$ by 4.2; hence $d(S t S)$ contains $[M(S), H t H]$ and thus $H s H$. Therefore $H s H \cap S t S \neq \varnothing$ and so $s \in S t S$, whence $s \leqslant_{0} t$.

LEMma 4.5. Let $S$ be a compact monoid in which $H s H=D(s)$ for all $s \in S$ (with $H=H(1)$ ). Then any surjective morphism $q: S \rightarrow \widetilde{S}$ maps $H$ onto $\widetilde{H}$, the group of units of $\widetilde{S}$. 
Proof. Trivially and without any hypothesis, $q$ maps $H$ into $\widetilde{H}$. The subset $S^{\prime}=q^{-1}(\widetilde{H})$ is a closed submonoid of $S$ with $q\left(S^{\prime}\right)=\widetilde{H}$, since $q$ is surjective. Let $M=M\left(S^{\prime}\right)$ be its minimal ideal and $e$ an idempotent in $M$. Then $q(e)$ is an idempotent in $\widetilde{H}$; hence $q(e)=1$. Since $M$ is a paragroup we must have $M \subseteq D(e)$ (where $D(e)$ denotes the $D$-class of $e$ in $S$ ). But $D(e)=\mathrm{HeH}$ by hypothesis, so $q(D(e)$ ) $=q(H e H)=q(H) q(e) q(H)=q(H) \subseteq \widetilde{H} ;$ hence $D(e) \subseteq q^{-1}(\widetilde{H})=S^{\prime}$, and thus $D(e)$ $=M$. By $\left[6,2.22\right.$, p. 24] we conclude $\widetilde{H}=q(M)$ from $\widetilde{H}=q\left(S^{\prime}\right)$. Therefore $\widetilde{H}$ $=q(M)=q(D(e))=q(H)$.

LemMA 4.6. Let $S$ be a TO-monoid and $q: S \rightarrow \widetilde{S}$ a surjective morphism. Then $\widetilde{S}$ is a TO-monoid.

Proof. Let $\widetilde{D}$ be the $D$-relation on $\widetilde{S}$. Then $q$ induces a monotone surjective map $q^{\prime}: S / D \rightarrow \widetilde{S} / \widetilde{D}$. Therefore $\widetilde{S} / \widetilde{D}$ is a totally ordered (compact connected) space. If $s \in S$ then the $D$-quasi-order induces on the compact space $q^{-1}(q(s))$ a total quasi-order with closed graph; let us pick for each $s$ a $D$-maximal element $s^{*} \in q^{-1}(q(s))$. Then $q\left(s^{*}\right)=q(s)$ and $D\left(s^{*}\right)=\max \{D(x) \mid q(x)=q(s)\}$. For two elements $s, t \in S$ we have $q(s) \widetilde{D} q(t)$ iff $\widetilde{S} q(s) \widetilde{S}=\widetilde{S} q(t) \widetilde{S}$ iff $S q^{-1}(q(s)) S=$ $S q^{-1}(q(t)) S$. In a TO-monoid every closed ideal is principal, since indeed it is generated by any element in its top D-class (4.4). Thus $S q^{-1}(q(s)) S=S s^{*} S$, whence $q(s) \widetilde{D} q(t)$ is equivalent to $S s^{*} S=S t^{*} S$, i.e., to $s^{*} D t^{*}$. By 4.3 , this means $H s^{*} H=H t^{*} H$, and by 4.5 this in turn implies $\widetilde{H} q(s) \widetilde{H}=\widetilde{H} q\left(s^{*}\right) \widetilde{H}=\widetilde{H} q\left(t^{*}\right) \widetilde{H}=$ $\widetilde{H} q(t) \widetilde{H}$ where $\widetilde{H}$ is the group of units of $S$. Thus, for every $\widetilde{s} \in \widetilde{S}$, we have $\widetilde{D}(\widetilde{S}) \subseteq \widetilde{H} \widetilde{S} \widetilde{H}$. The converse inclusion $\widetilde{H} \widetilde{s} \widetilde{H} \widetilde{D}(\widetilde{S})$ is always true. Hence $\widetilde{D}(\widetilde{S})$ $=\widetilde{H} \widetilde{S} \widetilde{H}$ for all $\widetilde{S} \in \widetilde{S}$. Thus the orbit space $\widetilde{S} /(\widetilde{H} \times \widetilde{H})$ of cosets $\widetilde{H} \widetilde{S} \widetilde{H}$ is a totally ordered ompact connected space since $\widetilde{S} / \widetilde{D}$ has these properties. This shows 4.1 (i) for $\widetilde{S}$.

Since $M(\widetilde{S})=\widetilde{D}(\widetilde{e})$ for some idempotent $\widetilde{e} \in M(\widetilde{S})$ the preceding shows $M(\widetilde{S})=\widetilde{H} \widetilde{e} \widetilde{H}$; since $q^{\prime}$ is monotone, it maps the smallest element $M(S)$ of $S$ into the smallest element of $\widetilde{S} / \widetilde{D}$, which is $M(\widetilde{S})$ (the order on $\widetilde{S} / \widetilde{D}$ being the $\widetilde{D}$-partial order). Hence 4.1 (ii) holds for $\widetilde{S}$, too.

LEMMA 4.7. Let $S$ be a compact monoid in which the component $S_{0}$ of 1 meets $M(S)$. If $M(S)=H m H$ for some $m \in M(S)$, then $E(M(S)) \subseteq Z\left(H_{0}, S\right)$, where $H_{0}=H(1)_{0}$.

Proof. By assumption we find an idempotent $e \in M(S) \cap . S_{0}=M\left(S_{0}\right)$. If $H m H=M(S)$ then we find $g, h \in H$ with $m=g e h$; hence $M(S)=M h M=$ $\mathrm{HgehH}=\mathrm{HeH}$. Now, if $(\mathrm{HeH})_{0}$ is the component of $\mathrm{HeH}$ containing e, $(\mathrm{HeH})_{0}$ $\subseteq S_{0}$, since $e \in S_{0}$, whence $(H e H)_{0} \subseteq M\left(S_{0}\right)$; but $M\left(S_{0}\right)$ contains $e$ and is a 
connected subspace of $\mathrm{HeH}$, so $M\left(\mathrm{~S}_{0}\right) \subseteq(\mathrm{HeH})_{0}$. Thus $M\left(\mathrm{~S}_{0}\right)=(\mathrm{HeH})_{0}$. We claim that $(\mathrm{HeH})_{0}=H_{0} e H_{0}$. Indeed the function $(g, h) \mapsto e \circ(g, h)=g^{-1} e h$ : $H \times H \rightarrow H e H$ factors through the quotient space $(H \times H) / K$ where $K=$ $\left\{(g, h) \mid g^{-1} e h=e\right\}$ is the isotropy group at $e$, and $\mathrm{HeH}$ is homeomorphic to this quotient space under $K(g, h) \rightarrow g^{-1} e h$. We use the following group theoretical

LemMA. If $G$ is a compact group, $B$ is a closed subgroup, $(G / B)_{0}$ the component of $B \in G / B$, and $G_{0}$ the identity component of $G$, then $(G / B)_{0}=G_{0} B / B$.

Thus $((H \times H) K / K)_{0}=\left(H_{0} \times H_{0}\right) K / K$, whence $(H e H)_{0}=H_{0} e H_{0}$, and so $M\left(S_{0}\right)=H_{0} e H_{0}$. We now apply $\left[6,3.1\right.$, p. 221] to $S_{0}$ and conclude that $M\left(S_{0}\right)$ $=H_{S_{0}}(e)$. Therefore $H_{0} e \cup e H_{0} \subseteq H(e)$, whence $h e=e h e=e h$ for all $h \in H_{0}$, i.e., $e \in Z\left(H_{0}, S\right)$. If $f \in E(M(S))$, then there are elements $g, h \in H$ with $f=g e h$; then $H_{0} f=H_{0} g e h=g H_{0} e h=g e H_{0} h=g e h H_{0}=f H_{0}$ since $H_{0}$ is normal in $H$ and $e$ commutes with $H_{0}$. Hence $f$ commutes with $H_{0}$ and thus $f \in Z\left(H_{0}, S\right)$.

Lemma 4.8. Let HeH be a subsemigroup of a TO-monoid. Then $d^{-1}[d(e), d(1)]$ is a TO-submonoid with minimal ideal HeH.

Proof. Suppose $e \leqslant s, t$. The left ideal $L=S s \cup M(S)$ is connected modulo $M(S)$ by 4.2. Similarly, $R / M(S)$ is connected, where $R=s S \cup M(S)$. The compact sets $d(L), d(R)$ then are connected, hence are intervals, both containing $M(S)$; but $M(S) \leqslant H e H \leqslant H s H$ and $M(S) \leqslant H e H \leqslant H t H$, so $S s \cap H e H \neq \varnothing$.

Therefore, for suitable elements $a, b \in \mathrm{HeH}$ we have $a \in S s$ and $b \in t S$ and thus $a b \in S s t S$, i.e., $a b \leqslant s t$. Since $\mathrm{HeH}$ is a subsemigroup we have $a b \in \mathrm{HeH}$, and so $D(e)=D(a b) \leqslant_{0} D(s t)$. This proves the assertion.

For purely technical reasons, let us say that a semigroup $S$ satisfies condition (Reg) iff

(Reg) Each regular $D$-class $D(e)$ is a subsemigroup.

The two preceding lemmas then yield immediately:

LEMMA 4.9. If a TO-monoid satisfies (Reg), then $E(S) \subseteq Z\left(H_{0}, S\right)$.

Lemma 4.10. If a TO-monoid $S$ satisfies (Reg), then $Z\left(H_{0}, S\right)_{0} \cap M(S) \neq \varnothing$.

Proof. By 4.4 the hypothesis (8) of 3.3 is satisfied. Because of 4.9, Proposition 3.7 applies with $G=H_{0}$ and proves the assertion.

This yields immediately

LEMMA 4.11. In a TO-monoid $S$ satisfying (Reg) the group $H_{0}$ is normal, i.e., $s H_{0}=H_{0} s$ for all $s \in S$.

Proof. Let $s \in S$; then $H s H=H(1)$ or $H s H=M(S)$ or $H s H$ separates the 
space between $M(S)$ and $H(1)$; hence $H s H \cap Z\left(H_{0}, S\right)_{0} \neq \varnothing$. Thus there are elements $z \in Z\left(H_{0}, S\right), g, h \in H$, with $s=g z h$. Then $H_{0} s=H_{0} g z h=g H_{0} z h=g z H_{0} h=g z h H_{0}$ $=s H_{0}$, since $H_{0}$ is normal in $H$ and $z \in Z\left(H_{0}, S\right)$.

By this result, the relation with cosets $H_{0} s$ is a congruence on $S$. We may therefore form the factor semigroup $S / H_{0}$. By Lemma 4.6 , the monoid $S / H_{0}$ is a TOsemigroup.

LEMMA 4.12. $S / H_{0}$ is one dimensional.

Proof. The group $\widetilde{H}$ of units of $\widetilde{S}=S / H_{0}$ is $H / H_{0}$, and thus is zero dimensional. Hence all $\widetilde{D}(\widetilde{S})=\widetilde{H} \widetilde{S} \widetilde{H}$ are zero dimensional as orbits under the action of a zero dimensional group. The orbit space $\widetilde{S} /(\widetilde{H} \times \widetilde{H})$ is compact connected and totally ordered, hence one dimensional. Thus by [1, Corollary 7.2, p. 143], we conclude $\operatorname{dim} \tilde{S}$ $=1$.

LEMMA 4.13. $Z(\widetilde{H}, \widetilde{S})_{0} \cap M(\widetilde{S}) \neq \varnothing$.

Proof. We assume $M(\widetilde{S})=0$ and show that $Z(\widetilde{H}, \widetilde{S})$ is connected; by the standard technique of forming the Rees quotient semigroup, this is no loss of generality. Now, there exists a unique normal $I$-semigroup $\widetilde{N}$ from 1 to 0 , i.e., an $I$-semigroup satisfying $\widetilde{N} \widetilde{s}=\widetilde{s} \widetilde{N}$ for all $\widetilde{s} \in \widetilde{S}$. (See [6, Theorem 4.10, p. 226].) In particular, $\widetilde{N}$ is invariant under inner automorphisms, but a compact automorphism group operates trivially on an $I$-semigroup $[6,3.5$, p. 334], whence $\widetilde{N} \subseteq$ $Z(\widetilde{H}, \widetilde{S})$.

LEMMA 4.14. $\widetilde{H}$ is normal in $\widetilde{S}$.

The proof is completely analogous to the proof of 4.11 .

This leads to the final lemma in the series:

LEMмA 4.15. In a TO-monoid satisfying (Reg), the group of units $H$ is normal; hence $Z(H, S)_{0} \cap M(S) \neq \varnothing$.

Proof. Let $s \in S$; then $\left(H / H_{0}\right)\left(H_{0} s\right)=\left(H_{0} s\right)\left(H / H_{0}\right)$ in $S / H_{0}$ by 4.14 . Hence $H s=H_{0} s H$. By 4.11 we have $H_{0} s=s H_{0}$, so $H s=s H_{0} H=s H$. The remainder follows by Theorem 3.1.

After we have pushed the development of TO-monoids to this point we can now apply the theory developed in [6, Chapter C, §2, p. $200 \mathrm{ff}$.]. The standard hypothesis there is that the space $S / G$ of cosets $G s$ modulo some fixed group $G$ of units is a totally ordered connected space. This is now satisfied with $G=H$. However, our present development is independent up to Propositions 2.8 and 2.9 on p. 204 in [6]. We will only use 2.9 and record it in the following 
LEM M A 4.16. Under the hypotheses of 4.15 , the submonoid $Z(H, S)_{0}$ contains an I-semigroup $N$ which contains the identity and meets $M(S)$.

In order to have a convenient formulation of our main result in this section we introduce the following

DEFINITION 4.17. A topological space is said to be totally ordered if there exists a total order on it such that the order topology agrees with the given one.

We can now formulate the principal result of this section:

THEOREM 4.18 (THE $H \times H$-THEOREM). Let $S$ be a compact monoid and $H=$ $H(1)$ its group of units. The following conditions are equivalent:

(1) The space $Y$ of double cosets $H s H, s \in S$, is connected and totally ordered in such a fashion that $M(S)=H m H$ for some $m \in M(S), M(S)$ is minimal in $Y$ and that, moreover, each regular D-class is a subsemigroup.

(2) The space $X$ of left cosets $H s, s \in S$, is connected and totally ordered in such a fashion that $M(S)=$ Hm for some $m \in M(S)$, and $M(S)$ is minimal in $X$.

(3) There is a central I-semigroup $N$ in $S$ such that $S=N H$.

REMARK. Condition (3) says in effect that there is a surjective morphism $(n, h) \mapsto n h: N \times H \rightarrow S$ of compact monoids whose kernel relation has the cosets $\{n\} \times H_{n} h$, where $\left\{H_{n} \mid n \in N\right\}$ is a family of closed normal subgroups of $H$ satisfying the conditions (a) $n<n^{\prime} \Rightarrow H_{n^{\prime}} \subseteq H_{n}$ and (b) $\bigcap\left\{H_{n} \mid n<n^{\prime}\right\}=H_{n^{\prime}}$ for all $n^{\prime} \in N$.

Proof OF 4.18. (1) $\Rightarrow$ (2). By 4.15 we have $H s H=H s$ for all $s \in S$. (2) $\Rightarrow$ (3). See [6, Theorem VIII, p. 204]. (3) $\Rightarrow$ (1) is trivial.

Note. In view of Lemma 4.16 we can prove directly $(1) \Rightarrow(3)$ using only [6, Proposition 2.9, p. 204] rather than the entire development of C-2 in [6].

The proof of the remark is an elementary exercise along the lines of the proof of $[6,2.1$, p. 84].

Corollary 3.6 above is an immediate consequence of the $H \times H$ Theorem 4.18, too, if one recalls that under the hypotheses of 3.6 all $D$-classes $D(s)$ equal $H s H$. There is a good likelihood that the somewhat extraneous hypothesis in 4.18(1) that all regular $D$-classes are subsemigroups is not actually necessary but, rather, turns out to be a consequence of the other assumptions. However, at this time we have been unable to overcome certain difficulties arising in this context. A similar phenomenon occurs in [6, Theorem VII, p. 196].

Let us, in conclusion, look back at the proof of the $H \times H$ Theorem 4.18 and pinpoint the deep and difficult steps. All lemmas through 4.6 use comparatively elementary arguments. In Lemma 4.7 one uses the first deep fact by referring to 3.1 on p. 221 of [6]. This result is based on the fact that a quotient space of a 
compact group which is acyclic in cohomology over the rationals and the integers modulo 2 in fact singleton (see [6, p. $306 \mathrm{ff}$.]). The next step of greater depth comes in Lemma 4.10 where we use Theorem 3.3 above, which is based on all of the developments of $\S \S 1$ and 2 and on the centralizing theorem for left normal groups of units [5]. Finally, if one were to track down the results used to establish 4.16, one would find that they are based eventually on a nontrivial proposition about cylindrical semigroups [6, Proposition 2.4, p. 88 ]

It should be observed, on the other side, that the centralizing theorem for connected abelian groups of units [6, Theorem I, p. 62] which is based on the full theory of actions of compact connected abelian groups on rationally acyclic spaces is used nowhere in this paper.

The significance of the class of semigroups characterized by 4.18 in a new fashion was extensively discussed in the exercises to C-2 in [6] .

5. Some examples and complementary results. We have settled the centralizing conjecture if $H$ is a congruence. If $L$ is a congruence, no complete answer is known; in fact the situation is not better here than in the case that $D$ is a congruence; in both of these situations we have partial results exemplified by our theorems in $\S \S 3$ and 4. It is instructive to see what kind of examples of compact monoids one might have in which $L$ is a congruence, and how close one might be able to come to a counterexample to the centralizing conjecture in this case. We therefore introduce a new class of compact monoids in this section.

We recall a class of congruences which occurred in $3.3(1)$ :

DEFINITION 5.1. We say that a group $G \subseteq H(1)$ of units in a monoid is dominated by a congruence $Q$ on $S$ if

(i) $G \subseteq Q(1)$ and

(ii) $Q \cap R \subseteq L$ for Green's relations $R$ and $L$.

Note that the full group $H(1)$ is dominated by $H$ and $L$, if these relations are congruences.

PROPOSITION 5.2. Let $S$ be a monoid and $G$ its group of units. Suppose that $Q$ is a congruence and $G$ is dominated by $Q$. Then:

(a) For each $s \in S$ the stability group $G_{S}=\{g \in G \mid s g=s\}$ is normal and thus is constant on orbits $s G$.

(b) For each $e \in E(S)$, the set eG is a subgroup of $H(e)$ which is isomorphic to $G / G_{e}$, and the set $G e G$ is a left group with $E(G e G)=e^{G}=\left\{\mathrm{geg}^{-1} \mid g \in G\right\}$ and maximal group $H_{G e G}(f)=f G$ for $f=g_{e g}^{-1}$. The map $(x, y) \mapsto x y ; e^{G} \times e G \longmapsto$ $\mathrm{GeG}$ is an isomorphism.

Proof. If $e$ is idempotent, then $e G \subseteq Q(e) Q(1) \subseteq Q(e)$, but also $e G \subseteq R(e)$; 
hence $e G \subseteq(Q \cap R)(e) \subseteq L(e)$. Now $e$ is a right identity for $L(e)$; thus $e g=$ $e g e$, whence $(e g)(e h)=e(g h)$; thus $g \mapsto e g: G \rightarrow e G$ is a homomorphism of $G$ into $H(e)$; hence $e G$ is a group and $G_{e}$, being the kernel of this morphism, is a normal subgroup. Clearly $(G e G)(G e G)=G(e G e) G=G(e G) G=G e G$ so $G e G$ is a semigroup.

Since $e G \subseteq L(e)$, then $G e G \subseteq G L(e)=L(e)$. Let $H(L(e))$ denote the union of all groups in $L(e)$; then $H(L(e))$ is a left group [3, Exercise 4, p. 66]. Let $e^{G}$ denote the set of conjugates $g e g^{-1}, g \in G$. Then $e^{G} \subseteq E(L(e)) \subseteq H(L(e))$, so $G e G=e^{G} G=e^{G} e G \subseteq H(L(e)) H(e) \subseteq H(L(e))$. Hence $G e G$, being a subsemigroup of a left group, is a left group. The map $e^{G} \times e G \rightarrow G e G$ given by $(x, y)$ $\mapsto x y$ is clearly surjective. If $(g e h)^{2}=g e h$, then $e=e g h e=e g h$, and thus $h \in$ $g^{-1} G_{e}=G_{e} g^{-1}$, whence $g e h=g e g^{-1}$. This shows $E(g e G)=e^{G}$. Since $H_{G e G}(e)=(G e G)=e G$, this map is in fact an isomorphism.

We have shown earlier $[5$, p. $40,5.1]$ how the situation of 5.2 may be realized. However, in the following we construct a new class of examples.

Let $X$ be a set and $L$ a subsemilattice of the power set $2^{X}$ relative to $U$. Let $\Gamma$ be a semigroup.

LEMMA 5.3. $\Gamma^{X} \times L$ is a subsemigroup under the multiplication $(f, A)(g, B)$ $=(f[A] g, A \cup B)$ where

$$
(f[A] g)(x)= \begin{cases}f(x) g & \text { if } x \in A, \\ f(x) g(x) & \text { if } x \notin A,\end{cases}
$$

and the projection $\lambda: \Gamma^{X} \times L \rightarrow L$ is a morphism.

Proof. We have to check that

$$
\begin{aligned}
\{(f, A)(g, B)\}(h, C) & =(f[A] g, A \cup B)(h, C) \\
& =((f[A] g)[A \cup B] h,(A \cup B) \cup C)
\end{aligned}
$$

equals

$$
\begin{aligned}
(f, A)\{(g, B)(h, C)\} & =(f, A)(g[B] h, B \cup C) \\
& =(f[A](g[B] h), A \cup(B \cup C)) .
\end{aligned}
$$

Thus we must check

$$
\text { (asso) } \quad(f[\mathrm{~A}] g)[A \cup B] h=f[A](g[B] h) .
$$

This is straightforward but tedious; an illustration by Venn diagrams may be helpful. It is obvious that $\lambda: \Gamma^{X} \times L \rightarrow L$ is a morphism.

Lemma 5.4. Suppose that $\Gamma$ is a group. Then $\lambda$ is equivalent to the 
L-quotient map; specifically, all $\Gamma \times\{A\}$ are left groups and L-classes. If $\Gamma^{A} \times \Gamma^{X \backslash A}$ is the left group obtained by giving $\Gamma^{A}$ the left zero multiplication and $\Gamma^{X}$ the pointwise group operation, then the function $\phi_{A}: \Gamma^{A} \times \Gamma^{X \backslash A} \rightarrow$ $\Gamma^{X} \times\{A\}$ given $\phi_{A}(f, g)=(h, A)$ with $h|A=f, h| X \backslash A=g$, is an isomorphism. It follows, in particular, that $L$ is a congruence.

PROOF. Since $\lambda$ is a morphism and $L$ is a semilattice, certainly $\lambda$ must collapse all $L$-classes. The remaining assertions are easily checked and yield that the inverse images of singletons under $\lambda$ are left groups, hence are $L$-classes.

LEMMA 5.5. Under the assumptions of Lemma 5.4, the H-class of $(f, a)$ is $\{(g, A)|g| A=f\} \cong \Gamma^{X \backslash A}$.

If $\phi^{\prime \prime} \in L$, then $\Gamma^{X} \times\{\phi\}$ is the group of units $H=H(1)$ and for each $s \in$ $S=\Gamma^{X} \times L$ we have $H s=L(s), s H=H(s)$. In particular, $H(1)$ is left normal. However $L(s)=H(s)$ implies $s \in H$.

Proof. Straightforward.

LEMMA 5.6. Under the assumptions of Lemma 5.4 if $\phi \in L$, then $Z(H, S)$ $=Z(H, H)=Z(\Gamma, \Gamma)^{X}$.

Now we topologize this example in various ways. First we produce a compact example. Let $X$ be a (discrete) set and give the power set $2^{X}$ the product topology (identifying $2^{X}$ with the functions into the two element max-semilattice $2=\{0,1\})$. Then $2^{X}$ is a compact, zero dimensional semilattice. Let $\Gamma$ be a compact group and give $\Gamma^{X}$ the product topology.

PROPOSITION 5.7. $\Gamma^{X} \times 2^{X}$ is a compact monoid. The connected component of $(f, A)$ is $\Gamma_{0}^{X} f \times\{A\}$, where $\Gamma_{0}$ is the identity component of $\Gamma$.

Proof. We interpret the elements of $2^{X}$ as the characteristic functions of subsets of $X$. Let $U \times V$ be a basic neighborhood of $(f, A)(g, B)=(f[A] g, A \cup$ $B$ ); we may assume that $V$ consists of all $C$ which agree with $A \cup B$ on a finite set $F=\left\{x_{1}, \cdots, x_{n}\right\}$ and that $U$ contains all functions $h: X \rightarrow \Gamma$ with $h\left(x_{k}\right) \in$ $(f[A] g)\left(x_{k}\right) W$ for all $k=1, \cdots, n$ and some identity neighborhood $W$ of $\Gamma$. Let $W^{\prime} \subseteq W$ be an identity neighborhood of $\Gamma$ such that $f\left(x_{k}\right) W^{\prime} g\left(x_{k}\right) W^{\prime} \subseteq$ $f\left(x_{k}\right) g\left(x_{k}\right) W$ for all $k$. Consider $U_{1}=\left\{(h, C) \mid h\left(x_{k}\right) \in f\left(x_{k}\right) W^{\prime}\right.$ for all $k$ and $C$ agrees with $A$ on $F\}, U_{2}=\left\{(h, C) \mid h\left(x_{k}\right) \in g\left(x_{k}\right) W^{\prime}\right.$ for all $k$ and $C$ agrees with $B$ on $F$. Then $U_{1}$ and $U_{2}$ are neighborhoods of $(f, A),(g, B)$, respectively, and we take $\left(f^{\prime}, A^{\prime}\right) \in U_{1}$ and $\left(g^{\prime}, B^{\prime}\right) \in U_{2}$. Then $A^{\prime}$ agrees with $A$ on $F, B^{\prime}$ agrees with $B$ on $F$; hence $A^{\prime} \cup B^{\prime}$ agrees with $A \cup B$ on $F$ and is, therefore, contained in $V$. Let $x_{k} \in F$. Then 


$$
\begin{aligned}
\left(f^{\prime}\left[A^{\prime}\right] g^{\prime}\right)\left(x_{k}\right) & =\left(f^{\prime}[A] g\right)\left(x_{k}\right) \\
& =\left\{\begin{array}{l}
f^{\prime}\left(x_{k}\right) \in f\left(x_{k}\right) W^{\prime} \subseteq f\left(x_{k}\right) W \quad \text { if } x \in A, \\
f^{\prime}\left(x_{k}\right) g^{\prime}\left(x_{k}\right) \in f\left(x_{k}\right) W^{\prime} g\left(x_{k}\right) W^{\prime} \in f\left(x_{k}\right) g\left(x_{k}\right) W \quad \text { if } x \notin A .
\end{array}\right.
\end{aligned}
$$

Thus $f^{\prime}\left[A^{\prime}\right] g^{\prime} \in U$. This shows $U_{1} U_{2} \in U \times V$, which proves the continuity of multiplication. The remainder is clear.

One may now form the cone semigroup

$$
T=\left(\left[\Gamma^{X} \times 2^{X}\right] \times I\right) /\left(\left(\Gamma^{X} \times 2^{X}\right) \times\{0\}\right)
$$

with an $I$-semigroup $I$ (such as the ordinary unit interval). This gives us the following example (taking for $X$ an infinite set and for $\Gamma$ a compact group with trivial center):

COROLlARY 5.8. There is a compact connected monoid $T$ with zero having the following properties:

(a) $L$ is a congruence and $H(1)$ is left normal.

(b) $Z(H(1), T)$ is an I-semigroup from 1 to 0.

(c) For all idempotents with the exception of 1 and $0, L(e)=H(1) e$ is a proper left group and properly contains $H(e)=e H(1)$.

For all of these $e$ we have $L(e) \cap Z(H(1), T)=\varnothing$.

(d) Every neighborhood of $H(1)$ contains regular L-classes $L(e)$ other than $H(1)$.

Finally let us add that there are connected (but no compact connected) topologies for some of the examples defined in 5.3. Indeed let $X$ be the unit interval $[0,1]$ and $L$ the $U$-semilattice of subsets $[0, a[, a \in X$. Let $\Gamma$ be the multiplicative group $\{-1,1\}$ of real numbers $1,-1$ and let $\Gamma^{X}$ denote the multiplicative group of all (equivalence classes modulo null-functions) of Lebesgue measurable functions from $X$ into $\Gamma$. If $L$ is given the topology making the bijection $a \mapsto[0, a[: X \rightarrow L$ a homeomorphism when $X$ is given the natural topology, and if $\Gamma^{X}$ is given the $L^{1}$-topology, then $\Gamma^{X} \times L$ is an arcwise connected monoid with group $\Gamma^{X} \times\{\varnothing\}$ of units and minimal ideal $\Gamma^{X} \times\{[0,1]\}$; the centralizer of the group of units is this group itself.

\section{REFERENCES}

1. G. E. Bredon, Sheaf theory, McGraw-Hill, New York, 1967. MR 36 \#4552.

2. J. F. Berglund and K. H. Hofmann, Compact semitopological semigroups and weakly almost periodic functions, Lecture Notes in Math., no. 42, Springer-Verlag, Berlin and New York, 1967. MR 36 \#6531.

3. A. H. Clifford and G. B. Preston, The algebraic theory of semigroups, Vol. 1, Math. Surveys, no. 7, Amer. Math. Soc., Providence, R. I., 1961. MR 24 \#A2627. 
4. K. H. Hofmann, Introduction to the theory of compact groups. II, Tulane University Lecture Notes, 1969.

5. K. H. Hofmann and M. Mislove, The centralizing theorem for left normal groups of units in compact monoids, Semigroup Forum 3 (1971), 31-42. MR 47 \#392.

6. K. H. Hofmann and P.S. Mostert, Elements of compact semigroups, Charles E. Merrill, Columbus, Ohio, 1966. MR 35 \#285.

7. B. Madison, Semigroups on coset spaces, Duke Math. J. 36 (1969), 61-63. MR $38 \# 5983$.

8. D. Montgomery and C. T. Yang, The existence of a slice, Ann. of Math. (2) 65 (1957), 108-116. MR 19, 291.

9. J. T. Rogers, Jr and J. L. Tollefson, Involutions on solenoidal spaces, Fund. Math. 73 (1971/72), 11-19. MR 45 \#5982.

DEPARTMENT OF MATHEMATICS, TULANE UNIVERSITY, NEW ORLEANS, LOUISIANA 70118 